\title{
Number of branches in diffusion-limited aggregates: The skeleton
}

\author{
Stefan Schwarzer, ${ }^{1,2}$ Shlomo Havlin, ${ }^{1,3}$ Peter Ossadnik, ${ }^{1,4}$ and H. Eugene Stanley ${ }^{1}$ \\ 1 Center for Polymer Studies and Department of Physics, \\ Boston University, Boston, MA 02215 \\ ${ }^{2}$ Laboratoire de Physique Mécanique des Milieux Hétérogènes, Ecole Supérieure de Physique et \\ Chimie Industrielles, \\ 75231 Paris, Cedex 05, France \\ 3 Department of Physics, Bar-Ilan University, Ramat-Gan, Israel \\ 4 Thinking Machines Corporation, c/o GMD, Schloss Birlinghofen, \\ Postfach 1316, 53757 St. Augustin, Germany
}

(May 14, 2019)

\begin{abstract}
We develop the skeleton algorithm to define the number of main branches $N_{b}$ of diffusion-limited aggregation (DLA) clusters. The skeleton algorithm provides a systematic way to remove dangling side branches of the DLA cluster and has successfully been applied to study the ramification properties of percolation. We study the skeleton of comparatively large $\left(\approx 10^{6}\right.$ sites $)$ off-lattice DLA clusters in two, three and four spatial dimensions. We find that initially with increasing distance from the cluster seed the number of branches increases in all dimensions. In two dimensions, the increase in the number of branches levels off at larger distances, indicating a fixed number of $N_{b}=7.5 \pm 1.5$ main branches of DLA. In contrast, in three and four dimensions, the skeleton continues to ramify strongly as one proceeds from the cluster center outward, and we find no indication of a constant number of
\end{abstract}


main branches. Likewise, we find no indication for a fixed $N_{b}$ in a study of DLA on the Cayley tree. In two dimensions, we find strong corrections to scaling of logarithmic character, which can help to explain recently reported deviations from self-similar behavior.

61.50.Cj, 05.40.+j, 64.60.Ak, 81.10.Jt

Typeset using REVTEX 


\section{INTRODUCTION}

The fractal nature of diffusion-limited aggregation has been established by a number of studies, and a theoretical framework is being developed [1,2]. In the initial paper written by Witten and Sander [1], power-law behavior of the two-point correlation functions was found. Later, Meakin [3] observed power-law scaling of the radius of gyration of DLA in two (2D), three (3D), and four dimensions (4D). Enhanced on- and off-lattice algorithms facilitated studies of larger aggregates (Meakin [4], Ball and Brady [5]) and a striking influence of the lattice structure on the structure of the aggregates was found [6]. Advanced off-lattice simulations by Ossadnik [7] grew clusters up to $M=5 \times 10^{7}$. All these studies indicate or confirm a fractal dimension of $d_{f}=1.715 \pm 0.004$ as reported by Tolman and Meakin [8]. Apart from the scaling of the radius of gyration with cluster mass, fractality is revealed in studies of the branching structure of the aggregates by, e.g., Alstrøm et al. [9], Hinrichsen et al. [10], Ossadnik [11], and more recently Yekutieli et al. [12]. Self-similarity has also been found in a study by Argoul et al. calculating the moments of the mass distribution of on-lattice clusters [13], by Barabási and Vicsek's mapping of the cluster perimeter to a self-affine surface [14], in investigations of the fjord geometry of DLA [15, 16], and many more (for reviews, see, e.g, 1722]).

However, almost as old as the model itself are reports about deviations from a simple statistically self-similar structure. In Refs. [5,23] lattice anisotropy and deposition habit effects on the structure of lattice clusters were studied, Meakin and Vicsek [24] found that the tangential and radial two-point-correlation functions in DLA display different exponents. In recent works however, Hegger and Grassberger 25] point out that off-lattice DLA in strip geometry displays a slow approach to local isotropy. Differences arise in the fractal dimensions measured in circular or strip geometry [26]. Simple self-similar scaling is not sufficient to fully characterize the mass distribution within a DLA cluster, as recognized by Meakin and Havlin [27], Vicsek, Family and Meakin [28], Amitrano, Coniglio, Meakin, and Zanetti [29], and Mandelbrot [30]. Mandelbrot examined the lacunarity [31,32] of off-lattice 
DLA and found a trend towards increasing compactness for increasing systems size which is not reflected in the measurements of the radius of gyration of the clusters.

In our study we will apply the "skeleton" algorithm [33, 34] to calculate the number of main branches $N_{b}$ in off-lattice DLA (Sec. [I). We obtain the skeleton of a DLA cluster by removal of all the small dangling side branches of the cluster (we define the skeleton in the following section). Only the main branches that reach out to a certain fraction of the cluster radius remain. For a self-similar, scale-invariant structure we expect that the large-scale properties, like the number of main branches of the cluster, are not altered under rescaling. Conversely, we take an increase or decrease of the number of main branches with increasing cluster size to suggest that the structure is not self-similar.

In $2 \mathrm{D}$, we find that significant finite size effects of logarithmic nature are present which may be related to some of the observations reporting deviations from simple self-similar behavior. Asymptotically however, the number of main branches of 2D DLA approaches a constant number (Sec. III). In contrast to the behavior in 2D, the number of branches of 3D and 4D DLA increases with cluster mass, indicating that these clusters may not be self-similar (Sec. [V).

\section{THE SKELETON}

DLA has a loopless tree structure [35]. This observation is apparent in simulations of off-lattice aggregates 36,37] where an incoming particle is added to the cluster when its distance to the cluster is below a specific sticking distance. Its "parent" particle is the one that it was closest to at the moment of incorporation into the cluster. The child-parent relationship allows us to uniquely assign a generation number or "chemical distance from the seed" to every cluster particle. To this end, we assign to the seed particle of the DLA cluster the number $\ell=0$. The children of the seed are assigned the chemical distance $\ell=1$; children of $\ell=1$ particles have chemical distance $\ell=2$. In general, each particle inherits the chemical distance of its parent and increases this number by one to find its own. 
The chemical distance turns out to be a very useful quantity in studies of the branching properties of DLA 911] and we will use it here to define the skeleton of DLA - closely following Ref. 33.

Roughly speaking, we obtain the skeleton by removal of all the small dangling side branches of the cluster. To this end we first identify all the sites that are tips of branches - those sites to which no other particle has been attached during the growth process. The $\ell$ value of each tip $\ell_{\text {tip }}$ is then passed back to its parent and grandparent and so forth until we hit an ancestor site which has more than one child. In this case, only the largest of the $\ell_{\text {tip }}$ values of the children is retained. We iterate this process until all cluster sites "know" their $\ell_{\text {tip }}$ value.

Now let us choose an arbitrary value $\ell_{c}$ less than the largest chemical distance $\Lambda$ of any site from the seed. Given $\ell_{c}$, we define as the skeleton those sites for which both $\ell_{\text {tip }} \geq \ell_{c}$ and $\ell \leq \ell_{c}$ holds. Thus, side branches which have not grown out to at least $\ell_{c}$ do not contribute to the skeleton.

In Fig. 1, we display the skeletons of two randomly selected growing DLA clusters at mass $M=5000,50000,500000$. In this figure, $\ell_{c}$ is chosen to be half the "chemical radius" $\Lambda / 2$ of the cluster. Note that the termination points of the skeleton are located almost on a circle, although we use the chemical and not the Euclidean distance from the seed to define the skeleton. This indicates that DLA grows radially outward without forming loops. Indeed, it has been found that the fractal dimension $d_{\min }$ of the path connecting the cluster seed to a given site is equal to 1 [35]. Here, $d_{\min }$ is defined by $\ell \sim r^{d_{\min }}$. In other words, for cluster sites at a given distance $r$ from the cluster seed, the average value $\langle\ell\rangle$ is proportional to $r,\langle\ell\rangle \sim r$. For the remainder of this study, we will consider $\ell$ and $r$ as equivalent.

One physical interpretation of the skeleton in DLA can be obtained if we consider the aggregate as a conductor situated between the grounded seed and a circular electrode (a sphere or hypersphere in $3 \mathrm{D}$ or $4 \mathrm{D}$, respectively) of "radius" $\ell_{c}$. Then the skeleton is the collection of paths that contribute to the current through the aggregate.

The skeleton as defined above has some desirable and some less desirable features. First, 
its definition is certainly simple, which is desirable. Second, we see in Fig. 11 that in an intermediate range of $\ell$ the number of branches in the skeleton is small, which is another desirable feature since we claim that the skeleton identifies the main branches of the DLA cluster. However, we see that close to $\ell_{c}$ a lot of branches appear. Their presence ultimately reflects our ignorance as to whether a specific side branch will keep growing or die out. Thus, these branches are a very "physical" property, but, nevertheless, an undesirable feature. We will address this problem by keeping $\ell_{c}$ a variable and systematically test the dependence of the skeleton calculations on this parameter.

We define $\lambda \equiv \ell_{c} / \Lambda$ and consider ensembles of DLA clusters for which the the value $\Lambda$ is fixed. For such an ensemble, we define $B(\lambda, \Lambda ; \ell)$ as the average number of sites in the skeleton at chemical distance $\ell$ from the cluster seed. Note that $B(\lambda, \Lambda ; \ell)$ is defined only for $\ell \leq \ell_{c}$.

Some general conclusions about properties of the DLA skeleton can be drawn from the definition and are visible in Fig. 11.

(i) For $\ell=0$, only the cluster seed contributes, thus $B(\lambda, \Lambda ; \ell=0)=1$ independent of spatial dimension $d$ and cluster size $\Lambda$.

(ii) Since $\ell \sim r$, and since DLA is essentially loopless so that overhangs can be neglected, we can determine the value $B\left(\lambda, \Lambda ; \ell=\ell_{c}\right)$ from the number of intersection points of a "cookie cutter" of radius corresponding to $\ell_{c}$ with the DLA cluster. As the analog of a cookie cutter in 3D we consider a sphere, and in $4 \mathrm{D}$ a hypersphere. The result is $B\left(\lambda, \Lambda ; \ell_{c}\right) \sim \ell_{c}^{\mathrm{d}_{\mathrm{f}}-1}$ where $d_{f}$ denotes the fractal dimension of DLA, which depends on the embedding dimension $d$. The exponent $d_{f}-1$ follows from the fact that the codimension of the intersection of two sets is obtained as the sum of the codimensions of the two intersecting sets.

(iii) The number of sites in the skeleton increases monotonically with $\ell$, i.e., $B\left(\lambda, \Lambda ; \ell^{\prime}\right) \geq$ $B(\lambda, \Lambda ; \ell)$ if $\ell^{\prime} \geq \ell$. Qualitatively we observe from Fig. 1 that the increase is slow in the vicinity of the cluster seed, but is followed by a precipitous increase close to $\ell_{c}$. 
(iv) For fixed $\ell$ and cluster "radius" $\Lambda$, the number of sites in the skeleton decreases monotonically when $\lambda$ is increased, because some branches that contribute to the skeleton at some value of $\ell_{c}$ fail to reach larger values. In the extreme case, when $\lambda=1$, we obtain $B(1, \Lambda ; \ell)=1$, independent of $\Lambda$ and $\ell$.

In the study of the skeleton of percolation clusters [33], a slow increase of the number of arms for small $\ell$ was found to be due to finite size corrections. Taking this into account, one obtains for small $\ell$ a constant number of branches. In analogy to the percolation example, we use finite-size scaling techniques to extract information about DLA from our 2D, 3D and 4D skeleton calculations, which we present in the following sections. The DLA results in finite dimensions will be compared to the skeleton of a random Cayley tree model, which can be considered to be the limit of infinite dimensionality of DLA [38].

\section{SKELETON OF 2D DLA}

In Fig. 2 we display $B(\lambda=0.5, \Lambda ; \ell)$ as a function of $\ell / \ell_{c}$ for $\Lambda=100,316,1000,3162$, and 5000 . For $\Lambda \leq 1000$, we obtain our data from 500 off-lattice clusters, for $\Lambda \geq 3162$, 114 clusters are averaged. The clusters are grown stepwise and growth is interrupted for the analysis when $\Lambda$ equals one of the above listed values. For $\Lambda=5000$, the cluster mass $M$ is typically $M=1-1.5 \times 10^{6}$ sites.

As a function of $\ell / \ell_{c}$ we find that after an initial short transient the central part of the data displays approximately linear behavior. The linear region widens when the cluster size $\Lambda$ increases. The approximate linear behavior terminates when $\ell / \ell_{c}$ approaches $\approx 0.3$. Beyond $\ell / \ell_{c} \approx 0.3, B(0.5, \Lambda ; \ell)$ increases sharply towards the final value $\sim \ell_{c}^{d_{f}-1}$. However, the linear region of the data tends to become flatter as we proceed to larger $\Lambda$. Since $B(\lambda, \Lambda)$ is monotonically increasing, the slope $\alpha(\lambda, \Lambda)$ in the linear region cannot be negative. Thus, asymptotically $\alpha(\lambda, \Lambda)$ can either approach 0 or assume a finite value $>0$. We will refer to the restricted range of approximately linear behavior as the "scaling regime" of the skeleton.

In order to distinguish between these two possibilities, we examine the dependence of 
$\alpha(\lambda, \Lambda)$ on system size, here characterized by $\Lambda$. To this end, we determine the slope $\alpha(\lambda, \Lambda)$ of a linear least squares fit,

$$
\ln B(\lambda, \Lambda ; \ell) \approx \alpha(\lambda, \Lambda) \ln \left(\frac{\ell}{\ell_{c}}\right)+\beta(\lambda, \Lambda),
$$

to $\ln B(\lambda, \Lambda ; \ell)$ vs $\ln \left(\ell / \ell_{c}\right)$ for data points satisfying $\ell \geq 10$ and $\ell / \ell_{c} \leq 0.3$. We use the assumption, familiar from the estimate of critical exponents from finite system size calculations (see, e.g. [39]), that $\alpha(\lambda, \Lambda)$ may be written as

$$
\alpha(\lambda, \Lambda)=\alpha_{\infty}(\lambda)+f(\lambda, \Lambda)+\ldots
$$

Here, the leading correction term $f(\lambda, \Lambda)$ to the asymptotic value $\alpha_{\infty}(\lambda)$ is typically either (i) a power law $\sim \Lambda^{-\gamma}$ or (ii) a logarithmic term, e.g., $\sim 1 / \ln \Lambda$. Both vanish for $\Lambda \rightarrow \infty$. The dots indicate corrections that decrease faster than $f(\lambda, \Lambda)$ as $\Lambda \rightarrow \infty$.

In Fig. 3a we plot $\alpha(\lambda ; \Lambda)$ vs $1 / \ln \Lambda$ for different values of $\lambda$. From the almost linear behavior of $\alpha(\lambda ; \Lambda)$ for large $\Lambda$ we conclude that the leading order correction term can be approximated by the reciprocal of $\ln \Lambda$ (ii), i.e.,

$$
f(\lambda, \Lambda)=A(\lambda) / \ln \Lambda .
$$

Here, $A(\lambda)$ denotes an amplitude which depends on $\lambda$ as evident from Fig. Ba. However, our calculations do not allow us to exclude the possibility that $f(\lambda, \Lambda)$ vanishes in the power-law fashion (i), although, in this case the characteristic power $\gamma$ must be small, $\gamma<0.2$. Larger values of $\gamma$ would lead to significant curvature in the large $\Lambda$ behavior of $\alpha(\lambda, \Lambda)$ [cf. Fig. $3 \mathrm{~b}]$.

From figure Ba we conclude that $\alpha_{\infty}(\lambda)=0$ for $0.3 \leq \lambda \leq 0.8$ by extrapolating the trend displayed by $\alpha(\lambda, \Lambda)$ for the $\Lambda$ range accessed by our simulation. Since for $\lambda \rightarrow 1$, $B(\lambda, \Lambda ; \ell)=1$ for all $\ell$, we expect this result to also hold if $\lambda>0.8$. For small $\lambda$, the skeleton probes the frozen region of the DLA cluster which effectively stopped growing [29], so that our results should be less effected by possible slow structural changes in the growth zone of the cluster [16,40,41]. Therefore, although the fit region of $B(\lambda, \Lambda ; \ell)$ becomes narrower, we assume that $\alpha_{\infty}(\lambda)=0$ extrapolates into the small $\lambda$ regime. 
The implications of $\alpha_{\infty}(\lambda)=0$ are intriguing. Asymptotically, we can assign a fixed number of main branches $N_{b}$ to 2D DLA growth. We deduce the actual number of these branches from the behavior of the intercepts $\beta(\lambda ; \Lambda)$ of the fit (11) in the "flat" region of the skeleton. In Fig. Bc $\beta(\lambda ; \Lambda)$ is plotted as a function of $1 / \ln \Lambda$ for several $\lambda$. The value of $\beta(\lambda ; \Lambda)$ slightly increases as the cluster size grows - corresponding to more main branches in larger systems. However, the increase levels off at about $\beta_{\infty}(\lambda)=2 \pm 0.2$, so that on average $N_{b}=\exp \left[\beta_{\infty}(\lambda)\right]=7.5 \pm 1.5$ main branches result.

\section{DLA IN HIGHER DIMENSIONS: 3 AND 4}

We also perform simulations of off-lattice DLA in 3D and 4D. Our findings are quite different from the 2D case, a result not surprising in the light of earlier work suggesting that $2 \mathrm{D}$ is a special case [42]. In particular, our calculations suggest that in contrast to the $2 \mathrm{D}$ case, where $\alpha_{\infty}(\lambda)=0$, in the $3 \mathrm{D}$ and $4 \mathrm{D}$ case $\alpha_{\infty}(\lambda)>0$. We discuss in the following the numerical data supporting this result.

\section{Skeleton of $3 D D L A$}

In Fig. 6 we display $B(\lambda=0.5, \Lambda ; \ell)$ for 3D DLA. Different curves are characterized by different values of $\Lambda$. The largest value of $\Lambda$ is 560 , which corresponds to DLA clusters with $M \approx 10^{6}$ particles. The skeletons of $450(\Lambda \leq 200)$ and $75(\Lambda>200)$ clusters have been averaged to obtain the displayed data.

The tendency for the central part of the plot to become flatter as $\Lambda$ increases is much less pronounced than in 2D. This observation is supported by Fig. 国a, which shows the $\Lambda$ dependence of $\alpha(\lambda ; \Lambda)$. We obtain the slope $\alpha(\lambda ; \Lambda)$ from a linear least square fit of $\ln B(\lambda, \Lambda ; \ell)$ in the region characterized by $\ell \geq 10$ and $\ell / \ell_{c}<0.3$. Like in $2 \mathrm{D}$, also in $3 \mathrm{D}$ the correction term is $\sim 1 / \ln \Lambda$ (2), and likewise we cannot exclude that the correction is of power-law type with a small exponent. However, unlike in 2D, in the 3D case the slopes corresponding to the small $\lambda$ values associated with the "frozen" region of the cluster $\alpha(\lambda ; \Lambda)$ 
extrapolate to a non-zero value, $\alpha_{\infty}(\lambda) \approx 0.2$. This value indicates that in $3 \mathrm{D}$ the number of branches of the skeleton increases monotonically in power-law fashion as a function of $\ell / \ell_{c}$ even in the limit of infinite cluster size.

\section{Skeleton of $4 D D L A$}

The skeletons of 50 off-lattice DLA clusters were averaged to obtain the data displayed in Fig. 6. The clusters with $\Lambda=190$ contain up to $\approx 500000$ particles. Similar to the 3D case, the slopes in the central part of the $B(\lambda, \Lambda ; \ell)$ data do not tend to become flat with increasing mass and this behavior is reflected in the $\Lambda$ dependence of $\alpha(\lambda ; \Lambda)$, which we display in Figs. Ta and b. The quality of the 4D calculations is much poorer than that of the $3 \mathrm{D}$ and $2 \mathrm{D}$ calculations. A finite value $\alpha_{\infty}(\lambda)$ is consistent with the two finite size scaling plots Figs. 7a and b. As in 3D, such a finite value indicates a power-law branching of the skeleton in the scaling regime as the cluster mass goes to infinity.

Moreover, if we consider the intersections $\beta(\lambda, \Lambda)$ of the straight line fits to the scaling

region we observe that they may diverge as $\Lambda \rightarrow \infty$. Such behavior would imply that $B(\lambda, \Lambda ; \ell)$ may not converge to a limit as $\Lambda \rightarrow \infty$. Thus we expect rather strong deviations from self-similar behavior in $4 \mathrm{D}$ and for $\Lambda \rightarrow \infty$ no self-similar limit as in the $2 \mathrm{D}$ case may exist.

\section{CAYLEY TREE MODEL}

The realization of the DLA growth model on a Cayley tree is considered to be the meanfield or infinite dimensionality limit of DLA. DLA on the Cayley tree has been formulated as follows [38]. To the cluster seed (shell 0 ), we links $z$ surface sites (shell 1 ) $-z \geq 2$ is the functionality of the model. In each growth step, we select (occupy) one of the surface sites randomly. We then create $z-1$ new (empty) surface sites and link them to the just occupied site. The shell number of the new empty sites is equal to the shell number of the 
occupied parent incremented by 1 . Note that all occupied sites have $z$ links to neighbor sites and that all empty sites have exactly 1 neighbor site.

Since new cluster sites are randomly picked from the set of all surface sites of the growing tree, we effectively disregard screening of the interior parts of the tree, which in finite dimensions is the essential mechanism to produce low dimensional fractal structures [43]. We have numerically grown clusters of this tree model with functionality $z=3,4$ and 5 as large as 32 shells. As a function of the shell number, the number of occupied sites grows exponentially 38. Similar to the case of 3D and 4D DLA, the skeleton shows no sign of saturation as a function of $\ell / \ell_{c}$. The increase seems to have even exponential character, with $\ln B(\lambda, \Lambda=32 ; \ell) \propto \ell / \ell_{c}$, and prefactors that depend on $z$ and $\lambda$.

This behavior is consistent with the situation in 3D and 4D DLA, where the skeleton as a function of $\ell / \ell_{c}$ also increases, albeit in a power-law fashion.

\section{DISCUSSION}

\section{A. Previous results on the number of stable branches of DLA}

The number of branches $N_{b}$ of DLA has been used as an important morphological characterization of the cluster [4,44,49]. In 2D using conformal mapping arguments, Ball 46] has proposed that the maximum number of stable branches $N_{\max }$ is related to the mass scaling dimension $D$ of the cluster by

$$
\left(N_{\max } / 2-1\right)(D-1)=1 .
$$

For $D \approx 1.7$ this relation predicts $N_{\max } \approx 4.9$. Similarly, Turkevich and Scher 44 have modeled the the tips of the cluster as the corners of a convex polygonal cluster envelope. The number $N$ of branches then determines the tip angles and those in turn the mass scaling dimension, namely, $D-1=N /(N+2)$. If one replaces $D-1$ from Eq. (4) and assumes $N=N_{\max }$, we obtain $N_{\max } \approx 4.8$, in agreement with the previous prediction. 
Both numbers seem to be significantly smaller than our finding $N_{b} 7.5 \pm 1.5$ in $2 \mathrm{D}$.t

It is not clear why $N_{b}$ should be different from $N_{\max }$ following from Eq. (四). On the one hand, if the actual number of branches is larger than the maximum number of stable ones then the competition among branches for the incoming flux of random walkers will cause branches to die (cf. [50]). If, on the other hand, the number of branches is too small, then new branches will be created because the fjords of the aggregate are not sufficiently screened to suppress growth. If, in fact, $N_{\max }$ and $N_{b}$ do not coincide, the reason could be that large branches may finally split, and one of the now too numerous arms will finally die out, but slowly enough to increase the number of branches apparent in the skeleton.

\section{B. Relation to "lacunarity" measurements on DLA}

Recently, Mandelbrot has investigated the lacunarity of off-lattice DLA [30]. To calculate the lacunarity of a cluster of span $\Lambda$, we (i) select the frozen interior region of the cluster (of span $k \Lambda, k<1$ ) and rescale all the particles coordinates into the unit circle, then (ii) center solid disks of radius $\epsilon \ll 1$ on the rescaled particle positions and (iii) determine the area fraction $f$ of the unit circle covered by the $\epsilon$ disks. For a "normal" fractal, one expects $f$ to saturate when $\Lambda$ increases and in this case to provide a measure for the lacunarity of the object. For DLA however, $f$ increases continuously with $\Lambda$. Mandelbrot argues accordingly that either DLA displays a "massive transient" or a "limitless drift" towards increasing compactness.

We argue that the logarithmic finite size corrections that we see in 2D in the approach of $B(\lambda, \Lambda ; \ell)$ to its asymptotics can explain the "massive transient" picture. As we have seen above, $B(\lambda, \Lambda ; \ell)$ increases with system size corresponding to structurally different clusters

\footnotetext{
${ }^{1}$ Concerning the Turkevich-Scher assumption of a convex polygonal cluster envelope one may argue that it is possible to conceive cluster envelopes with more acute angles and a larger number of arms - possibly around 7 .
} 
with more main branches $N_{b}$. Consequently, the area fraction $f$ increases.

Thus, off-lattice DLA shows a very slow structural change towards its asymptotic structure, similar to its on-lattice siblings.

\section{Why is 2D DLA different from higher dimensional DLA?}

The remaining open question is why on the one hand $\alpha_{\infty}(\lambda) \rightarrow 0$ in $2 \mathrm{D}$, but, on the other hand, $\alpha_{\infty}(\lambda)>0$ in $3 \mathrm{D}$ and $4 \mathrm{D}$ and in the latter case possibly even a divergence of $\beta(\lambda, \Lambda)$ with $\Lambda$. Let us first note, that $2 \mathrm{D}$ is known to be a "critical dimension" for radial DLA with respect to the scaling properties of the growth probabilities of the surface sites 42, 51,52. We find two more indications supporting our result, one from a simple consideration of the cluster density in different spatial dimensionalities, the other from a comparison of the correlated DLA tree to a random tree model, for which the scaling behavior of the skeleton is known 34$]$.

\section{Cluster "density" and screening}

We have already noted above that the number of branches in the skeleton at its termination points at $\ell_{c}$ is $\sim \ell_{c}^{d_{f}-1}$. Thus, for given $\ell=\ell_{c}$, there are many more branches present in the $3 \mathrm{D}$ and $4 \mathrm{D}$ skeleton than in the $2 \mathrm{D}$ skeleton. In order that these branches contribute to the skeleton in the scaling region they must originate deep in the cluster interior.

With increasing dimension, the screening of the cluster interior from incoming particles decreases. This decrease manifests itself for example in the strong semi-exponential screening of the cluster fjords in 2D [53 57] in contrast to the power-law type 42] screening in 3D or in the screening free Cayley-tree model — where we also observe $\alpha_{\infty}(\lambda)>0$. Qualitatively, the reduction of screening can also be seen in the mean-field expression for the particle

penetration depth $\sim \rho^{-1} \sim \Lambda^{d-d_{f}}$. The penetration depth increases with $d$, because $d_{f}$ 
approaches $d-1$ from above. I $^{2}$

Therefore we conclude that the weaker screening in dimensions $d>2$ is responsible for the further increase in the number of branches of the skeleton both as a function of $\ell / \ell_{c}$ and as a function of cluster size $\Lambda$.

\section{Comparison to a random tree model}

DLA has the structure of a tree. Thus, we find it instructive to compare our findings for the off-lattice DLA skeleton to results for the skeleton of a random tree model [34]. This tree model is defined on a 2D square lattice and characterized by a tunable "intrinsic" dimension $d_{\ell}$, which determines how the mass $M(\ell)$ of the tree object depends on the chemical distance $\ell$ from the cluster seed, i.e., $M(\ell) \propto \ell^{d_{\ell}}$. To this end, we occupy randomly exactly $N(\ell)=\ell^{d_{\ell}-1}$ sites in the $\ell$ th chemical shell (or fewer if necessary) - all the other sites are blocked. Only those sites in the $\ell$ th shell shall be occupied that do not close loops, i.e., that are not neighbors to sites in previous shells. Note that apart from possible constraints due to the embedding lattice at small $\ell$, the topological structure of the clusters does not depend on the dimension of the embedding lattice.

Reference [34] then considers the skeleton $B(1, \Lambda ; \ell)$ of a tree with intrinsic dimension $d_{\ell}$. In contrast to DLA, here in general $B(1, \Lambda ; \ell) \geq 1$, because the last chemical shell contains $N(\Lambda)=\ell^{d_{\ell}-1} \gg 1$ sites. In these random tree models there exists a sharp transition value $d_{\ell}^{c}=1.65 \pm 0.05$ which separates two regions with different types of behavior. For trees with $d_{\ell}<d_{\ell}^{c}$ the skeleton displays a flat — approximately constant — region as a function of $\ell$, like the one observed in the 2D DLA case (non-branching skeleton). However, for larger values of $d_{\ell}$ the skeleton displays a well defined power-law increase as function of $\ell$, i.e., $\alpha_{\infty}(1)>0$ (branching skeleton).

\footnotetext{
${ }^{2}$ It is however known from simulations that at least in $2 \mathrm{D}$ this relation does not hold and the penetration depth is $\sim \Lambda$.
} 
Thus, if we consider DLA as such a random tree, we expect a branching skeleton for spatial dimension $d>2$, since $d_{\ell}=d_{f}>d_{\ell}^{c}$. In $2 \mathrm{D}, d_{f}$ is only slightly larger than $d_{\ell}^{c}$, or even equal to $d_{\ell}^{c}$ within the error bars, which is consistent with the observed non-branching skeleton.

\section{SUMMARY}

We have determined the skeleton of comparatively large $\left(\approx 10^{6}\right.$ sites $)$ off-lattice DLA clusters in 2D, 3D and 4D. We find that, asymptotically, in 2D the skeleton of DLA suggests a fixed number of $N_{b} \approx 7.5 \pm 1.5$ main branches and a self-similar structure. In 3D and $4 \mathrm{D}$, and possibly all spatial dimensions $d>4$, the DLA skeleton is a ramified object, which displays branching over the whole range of $\ell$-values for which it is defined.

For all dimensions we find strong finite-size effects corresponding to a slow change in the structure of DLA as the cluster size increases. The presence of strong corrections to scaling of logarithmic character in 2D DLA is in agreement with findings of deviations from self-similar behavior reported elsewhere.

Moreover, in $4 \mathrm{D}$, it is possible that the structure of the aggregate keeps changing even asymptotically, such that there is no self-similar limit.

\section{ACKNOWLEDGMENTS}

We are grateful for enlightening discussions with Z. Alexandrowicz, A.-L. Barabási, S.V. Buldyrev, A. Coniglio, M.F. Gyure, U. Essmann, H.J. Herrmann, J. Lee, M. Ossadnik, G. Peng, S. Prakash and S. Sastry. We also kindly acknowledge financial support by the NSF and benefits from computational resources at the HLRZ, KFA Jülich, Germany. S.S. is grateful to the scientific council of the NATO for financial support (granted through the DAAD, Bonn). 


\section{REFERENCES}

[1] T. A. Witten and L. Sander, Phys. Rev. Lett. 47, 1400 (1981).

[2] L. Pietronero, Rev. Mod. Phys. (1995, in press), and references therein.

[3] P. Meakin, Phys. Rev. A 27, 604 (1983).

[4] P. Meakin, H. E. Stanley, A. Coniglio, and T. A. Witten, Phys. Rev. A 32, 2364 (1985).

[5] R. C. Ball and R. M. Brady, J. Phys. A: Math. Gen. 18, L809 (1985).

[6] R. C. Ball, R. M. Brady, G. Rossi, and B. R. Thompson, Phys. Rev. Lett. 55, 1406 (1985).

[7] P. Ossadnik, Physica A 195, 319 (1993).

[8] S. Tolman and P. Meakin, Physica A 158, 801 (1989).

[9] P. Alstrøm, P. Trunfio, and H. E. Stanley, in Random Fluctuations and Pattern Growth: Experiments and Models, edited by H. E. Stanley and N. Ostrowsky (Kluwer Academic Publishers, Dordrecht, 1988), pp. 340-342.

[10] E. L. Hinrichsen, K. J. Måløy, J. Feder, and T. Jøssang, J. Phys. A: Math. Gen. 22, L271 (1989).

[11] P. Ossadnik, Phys. Rev. A 45, 1058 (1992).

[12] I. Yekutieli, B. B. Mandelbrot, and H. Kaufman, J. Phys. A: Math. Gen. 27, 275 (1994).

[13] F. Argoul, A. Arneodo, G. Grasseau, and H. L. Swinney, Phys. Rev. Lett. 61, 2558 (1988).

[14] A.-L. Barabási and T. Vicsek, Phys. Rev. A 41, 6881 (1990).

[15] A.-L. Barabási and T. Vicsek, J. Phys. A: Math. Gen. 23, L729 (1990).

[16] S. Schwarzer, S. Havlin, and H. E. Stanley, Phys. Rev. E 49, 1182 (1994). 
[17] P. Meakin, in Phase Transitions and Critical Phenomena, edited by C. Domb and J. L. Lebowitz (Academic, Orlando, 1988), Vol. 12.

[18] Fractals and Disorder, edited by A. Bunde (North-Holland, Amsterdam, 1992) [Proc. of the International Conference on Fractals and Disordered Systems, Hamburg, July 1992].

[19] J. Feder, Fractals (Pergamon, New York, 1988).

[20] Fractals: Physical Origin and Properties, edited by L. Pietronero (Plenum Publishing Co., London, 1990) [Proc. 1988 Erice Workshop on Fractals].

[21] On Growth and Form, edited by H. E. Stanley and N. Ostrowsky (Martinus Nijhoff Publishers, Boston, Dordrecht, 1986).

[22] T. Vicsek, Fractal Growth Phenomena, 2nd ed. (World, Singapore, 1992).

[23] P. Meakin, R. C. Ball, P. Ramanlal, and L. M. Sander, Phys. Rev. A 35, 5233 (1987).

[24] P. Meakin and T. Vicsek, Phys. Rev. A 32, 685 (1985).

[25] R. Hegger and P. Grassberger, Phys. Rev. Lett. 73, 1672 (1994).

[26] C. Evertsz, J. Phys. A: Math. Gen. 22, L1061 (1989).

[27] P. Meakin and S. Havlin, Phys. Rev. A 36, 4428 (1987).

[28] T. Vicsek, F. Family, and P. Meakin, Europhys. Lett. 12, 217 (1990).

[29] C. Amitrano, A. Coniglio, P. Meakin, and M. Zannetti, Phys. Rev. B 44, 4974 (1991).

[30] B. B. Mandelbrot, Physica A 191, 95 (1992).

[31] B. B. Mandelbrot, The Fractal Geometry of Nature (Freeman, New York, 1982).

[32] C. Allain and M. Cloitre, Phys. Rev. A 44, 3552 (1991).

[33] S. Havlin, R. Nossal, B. Trus, and G. Weiss, J. Phys. A: Math. Gen. 17, L957 (1984). 
[34] S. Havlin, R. Nossal, and B. Trus, Phys. Rev. A 32, 3829 (1985).

[35] P. Meakin, I. Majid, S. Havlin, and H. E. Stanley, J. Phys. A: Math. Gen. 17, L975 (1984).

[36] S. Tolman and P. Meakin, Phys. Rev. A 40, 428 (1989).

[37] P. Ossadnik, Physica A 176, 454 (1991).

[38] J. Vannimenus, B. Nickel, and V. Hakim, Phys. Rev. B 30, 391 (1984).

[39] K. Binder, Monte Carlo Methods in Statistical Physics, Vol. 7 of Topics in current physics, 2nd ed. (Springer, New York, Berlin, Heidelberg, 1986).

[40] M. Plischke and Z. Rácz, Phys. Rev. Lett. 53, 415 (1984).

[41] P. Ossadnik and J. Lee, J. Phys. A: Math. Gen. 26, 6789 (1993).

[42] S. Schwarzer, M. Wolf, S. Havlin, P. Meakin, and H. E. Stanley, Phys. Rev. A 46, R3016 (1992).

[43] R. Ball and T. A. Witten, Phys. Rev. A 29, 2966 (1984).

[44] L. A. Turkevich and H. Scher, Phys. Rev. Lett. 55, 1026 (1985).

[45] P. Meakin, A. Coniglio, H. E. Stanley, and T. A. Witten, Phys. Rev. A 34, 3325 (1986).

[46] R. C. Ball, Physica A 140, 62 (1986).

[47] T. C. Halsey, P. Meakin, and I. Procaccia, Phys. Rev. Lett. 56, 854 (1986).

[48] F. Argoul, A. Arneodo, J. Elezgaray, G. Grasseau, and R. Murenzi, Phys. Rev. A 41, 5537 (1990).

[49] A. Arneodo, E. Bacry, J. Muzy, and M. Tabard, Phys. Rev. Lett. 68, 3456 (1992).

[50] T. C. Halsey and M. Leibig, Phys. Rev. A 46, 7793 (1992).

[51] H. E. Stanley, A. Coniglio, S. Havlin, J. Lee, and S. Schwarzer, in On Clusters and 
Clustering, Random Materials and Processes, edited by P. J. Reynolds (North-Holland, Amsterdam, 1993), pp. 345-356.

[52] H. E. Stanley, A. Coniglio, S. Havlin, J. Lee, S. Schwarzer, and M. Wolf, Physica A 205, 254 (1994) [Proc. International Conference on Statistical Mechanics, Taipeh, 1993].

[53] J. Lee and H. E. Stanley, Phys. Rev. Lett. 62, 2945 (1988).

[54] S. Schwarzer, J. Lee, A. Bunde, H. E. Roman, S. Havlin, and H. E. Stanley, Phys. Rev. Lett. 65, 603 (1990).

[55] B. B. Mandelbrot and C. J. G. Evertsz, Physica A 177, 386 (1991).

[56] M. Wolf, Phys. Rev. A 43, 5504 (1991).

[57] M. Wolf, Phys. Rev. E 47, 1448 (1993). 


\section{FIGURES}

FIG. 1. Skeleton for 2 growing DLA clusters: $(a-c)$ first cluster, $(d-f)$ second cluster. Growth of the cluster has been interrupted at cluster masses $M=5000$ (a,d), 50000 (b,e) or 500000 (c,f). The determination of the skeleton is based on a value of $\ell_{c} / \Lambda=0.5$. The skeleton is then rescaled so that independent of cluster mass the same size results.

FIG. 2. Log-log plot of the $2 \mathrm{D}$ skeleton $B(\lambda=0.5, \Lambda ; \ell)$ vs $\ell / \ell_{c}$ for different $\Lambda$ values as indicated in the legend. For $\Lambda \leq 1300$ data are averaged over 850 off-lattice DLA, for $\Lambda>1300$ averages over 114 clusters are taken. For comparison, we have also averaged data in the constant $M$ ensemble, instead of the constant $\Lambda$ ensemble used here, and obtain similar results (not shown). Note that in an intermediate region of the plot $\left(\ell / \ell_{c}<0.3\right)$ a slope can be associated with $B(\lambda=0.5, \Lambda ; \ell)$ vs. $\ell / \ell_{c}$. As $\Lambda$ increases, this slope becomes increasingly smaller, indicating that the asymptotic curve may be flat in this "scaling" region. Inset: The number of branches in the skeleton at $\ell=\ell_{c}, B\left(\lambda, \Lambda ; \ell_{c}\right)$ is equal to the number of intersection points of a cookie cutter

of radius $\ell_{c}$ in $\ell$ space with the cluster, i.e., $B\left(\lambda, \Lambda ; \ell_{c}\right) \sim \Lambda^{d_{f}-1}$. The solid line is a guide to the eye and indicates a slope of $d_{f}-1=0.70$. Different symbols refer to different $\lambda=0.8(\bigcirc), 0.6(\square)$, $0.5(\triangle), 0.4(\nabla), 0.2(\bullet)$.

FIG. 3. Finite size behavior of the slopes $\alpha(\lambda, \Lambda)$ of $B(\lambda=0.5, \Lambda ; \ell)$ vs $\ell / \ell_{c}$ calculated from data in the range $\ell>10$ and $\ell / \ell_{c}<0.3$. The slopes $\alpha(\lambda, \Lambda)$ for different $\lambda$ as indicated in the legend of the figure are plotted vs (a) $1 / \ln \Lambda$ and (b) $1 / \Lambda$. In (a) the slopes lie on asymptotically straight lines indicating a finite size correction term $\sim 1 / \ln \Lambda$. This behavior should be contrasted to the calculations in (b) where the correction term is assumed to be proportional to $1 / L$ and pronounced curvature is displayed as $\Lambda$ increases. (c) Intercepts $\beta(\lambda, \Lambda)$ of the straight line fit with the ordinate vs $1 / \ln \Lambda$. If $\alpha_{\infty}(\lambda)=0$, then $\beta_{\infty}(\lambda)$ denotes the logarithm of the number of arms $N_{b}$ in the "flat" region of the skeleton. From $\beta_{\infty} \approx 2 \pm 0.2$ we conclude that $N_{b}=7.5 \pm 1.5$. 
FIG. 4. Log-log plot of the 3D skeleton $B(\lambda=0.5, \Lambda ; \ell)$ vs $\ell / \ell_{c}$ for different $\Lambda$ values as indicated in the legend. We take averages over $75(\Lambda>200)$ and $450(\Lambda \leq 200)$ clusters. Note that the tendency of the power-law region in the plot to become flatter with increasing $\Lambda$ is much less pronounced than in the 2D case 2 and is in fact rather indicating a non-zero $\alpha_{\infty}(\lambda)>0$. Inset: Number of branches in the skeleton at $\ell=\ell_{c}$. We expect $B\left(\lambda, \Lambda ; \ell_{c}\right) \sim \Lambda^{d_{f}-1}$. The solid line is a guide to the eye and indicates a slope of $1.5 \approx d_{f}-1$ in $3 \mathrm{D}$. Different symbols refer to different $\lambda$ and are the same as in Fig. 2.

FIG. 5. Finite size behavior of the slopes $\alpha(\lambda, \Lambda)$ of $B(\lambda=0.5, \Lambda ; \ell)$ vs $\ell / \ell_{c}$ calculated from data in the range $\ell>10$ and $\ell / \ell_{c}<0.3$ for 3D DLA. The slopes $\alpha(\lambda, \Lambda)$ for different $\lambda$ with symbols as in Fig. 3 are plotted vs (a) $1 / \ln \Lambda$ and (b) $1 / \Lambda$. (c) Intercepts $\beta(\lambda, \Lambda$ ) of the straight line fit with the ordinate vs $1 / \ln \Lambda$.

FIG. 6. Log-log plot of the $4 \mathrm{D}$ skeleton $B(\lambda=0.5, \Lambda ; \ell)$ vs $\ell / \ell_{c}$ for different $\Lambda$ values as indicated in the legend. As in the $3 \mathrm{D}$ case, the central region of the plot does not display a tendency to become flat as $\Lambda$ increases. However, the system sizes seem still too small to draw a final conclusion, in particular if one considers the size dependence of the slopes in the power-law region and the comparatively poorer quality of the calculations (see Fig. 7). Inset: Number of branches in the skeleton at $\ell=\ell_{c}$. We expect $B\left(\lambda, \Lambda ; \ell_{c}\right) \sim \Lambda^{d_{f}-1}$. The solid line is a guide to the eye and indicates a slope of $2.2 \approx d_{f}-1$ in $4 \mathrm{D}$. Different symbols refer to different $\lambda$ and are the same as in Fig. 2.

FIG. 7. Finite size behavior of the slopes $\alpha(\lambda ; \Lambda)$ of $B(\lambda=0.5, \Lambda ; \ell)$ vs $\ell / \ell_{c}$ calculated from data in the range $\ell>10$ and $\ell / \ell_{c}<0.3$ for $4 \mathrm{D}$ DLA. The slopes $\alpha(\lambda, \Lambda)$ for different $\lambda$ with symbols as in Fig. 3 are plotted vs (a) $1 / \ln \Lambda$ and (b) $1 / \Lambda$. (c) Intercepts $\beta(\lambda, \Lambda$ ) of the straight line fit with the ordinate vs $1 / \ln \Lambda$. 

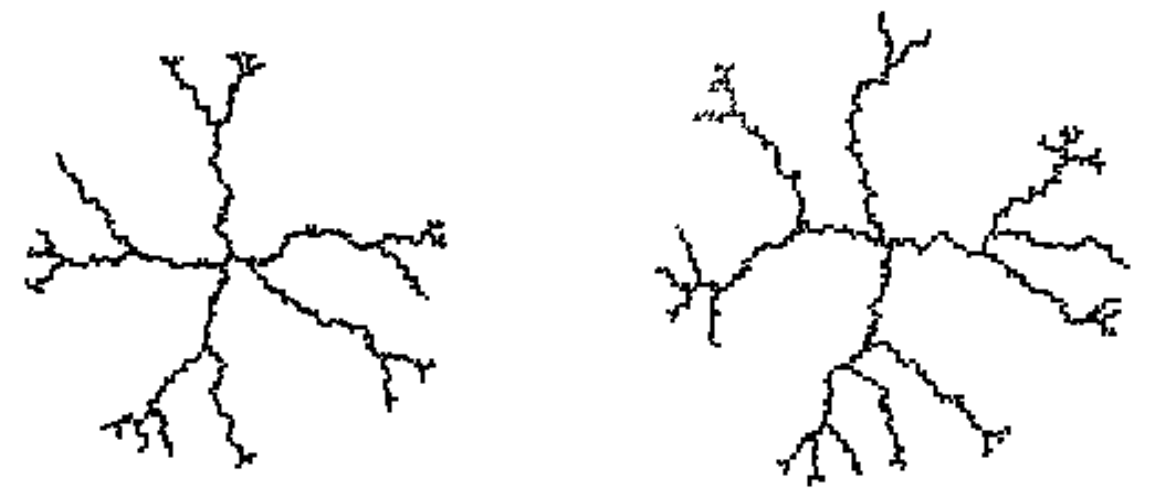

(a)

(d)
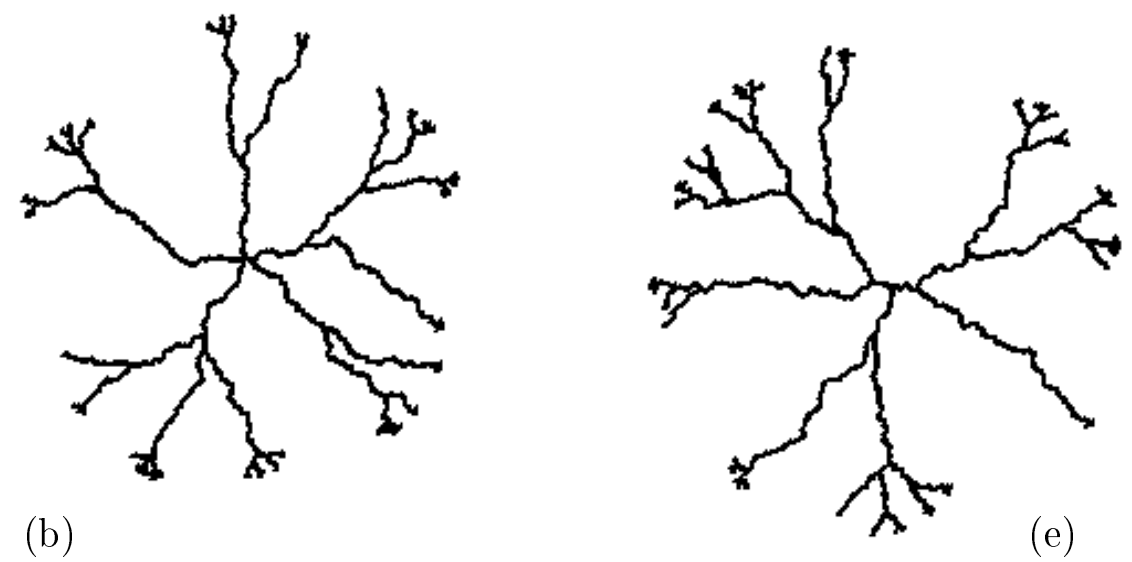

(b)
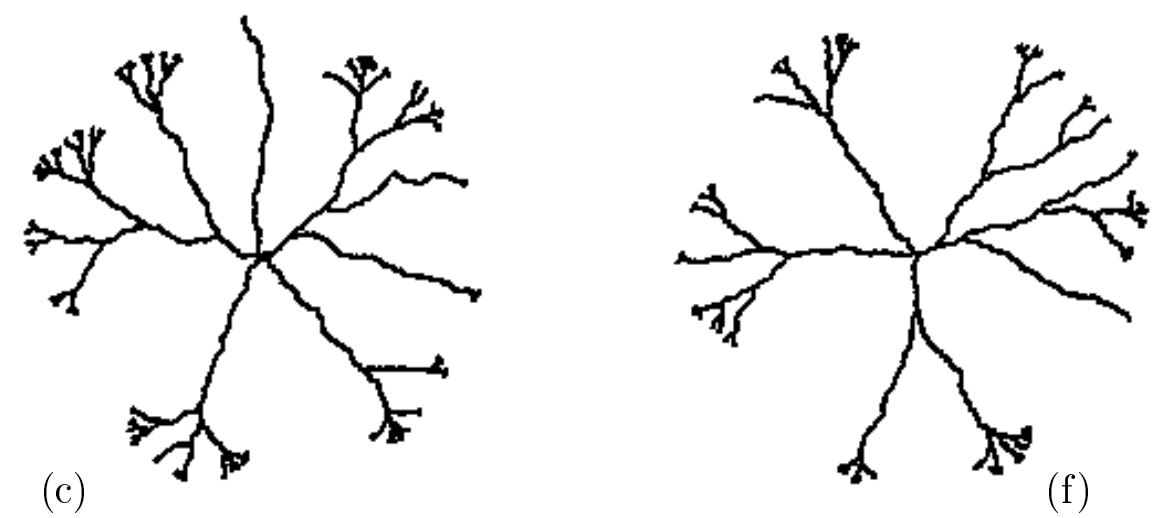

(f) 


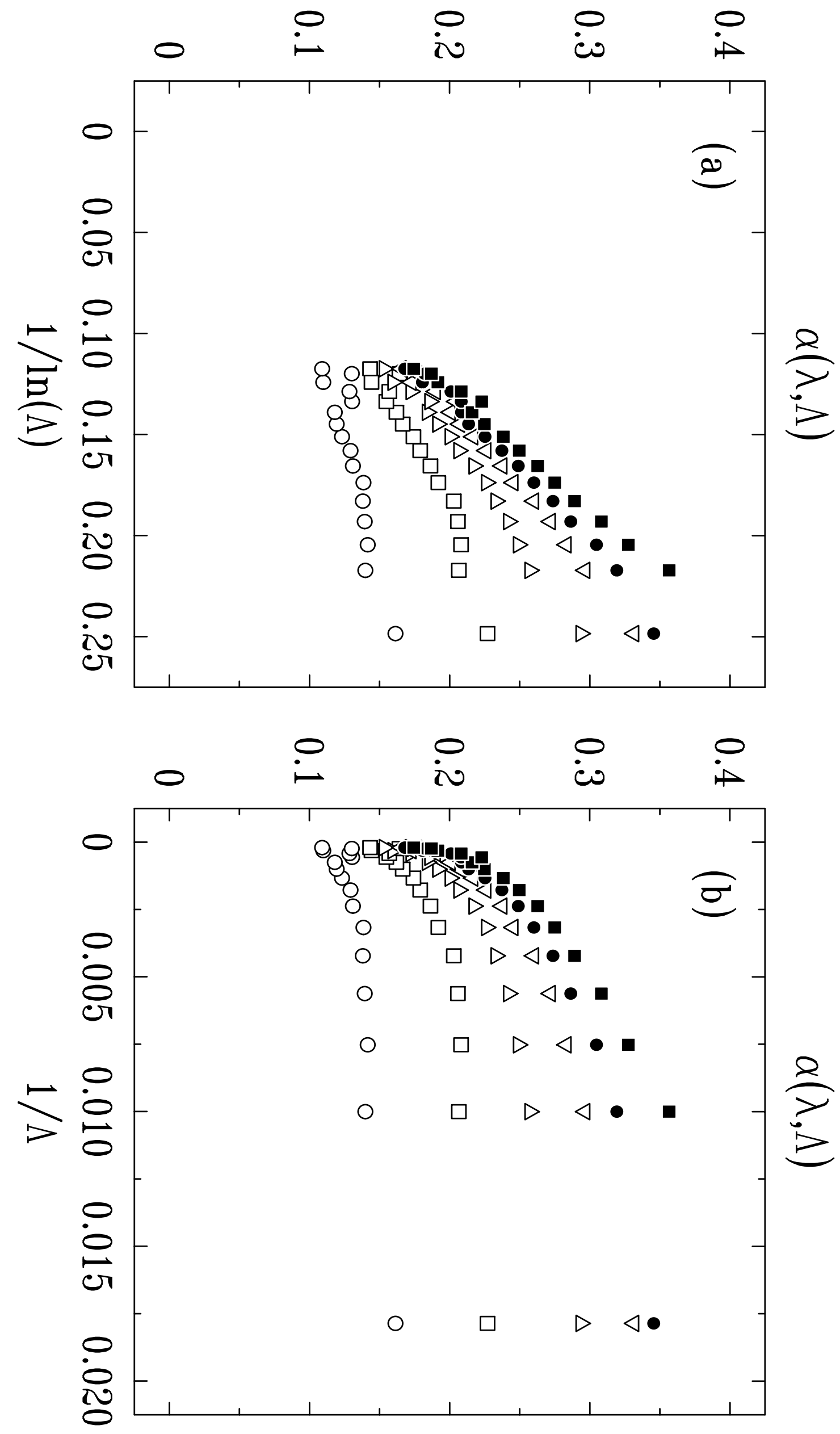




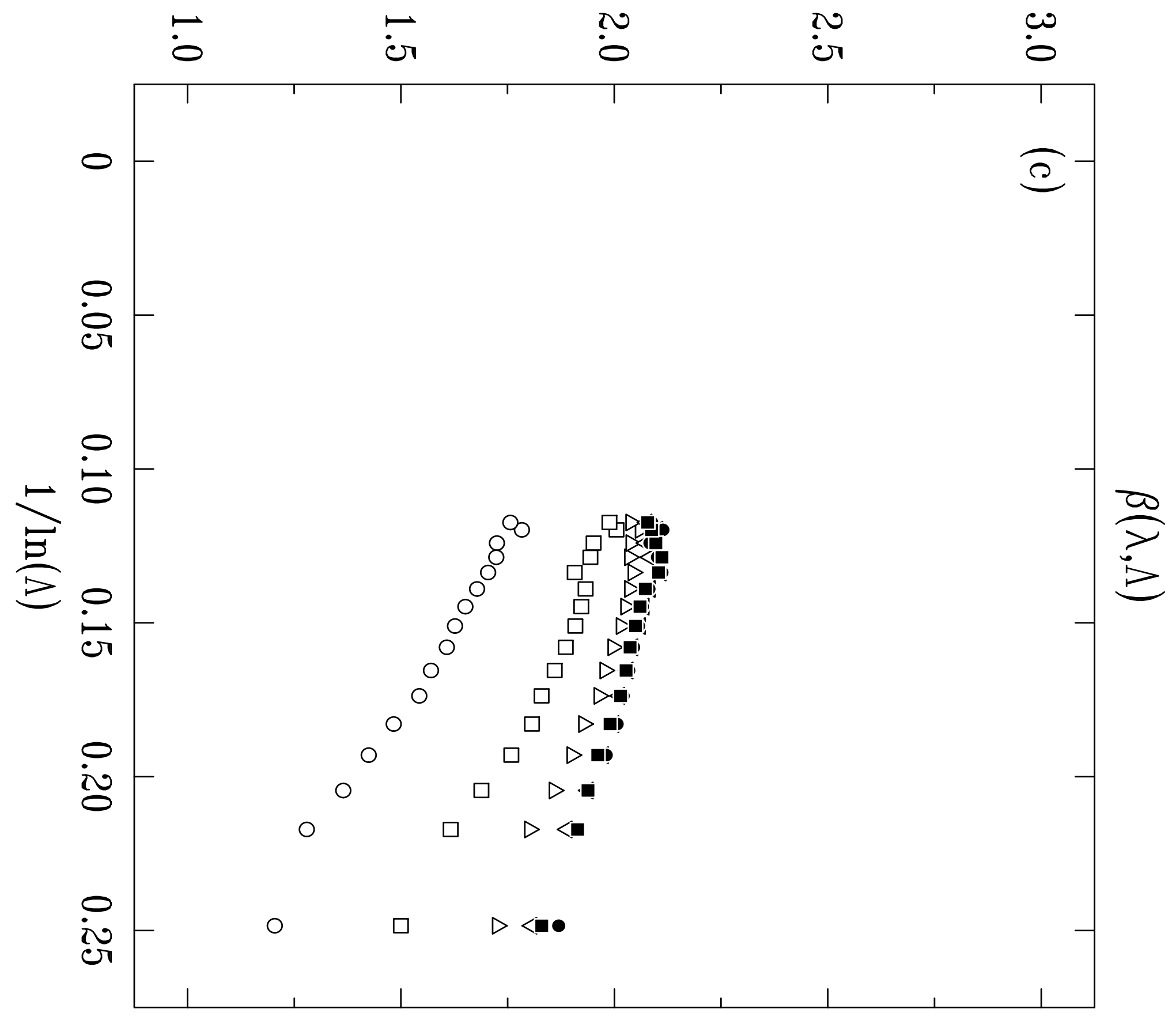




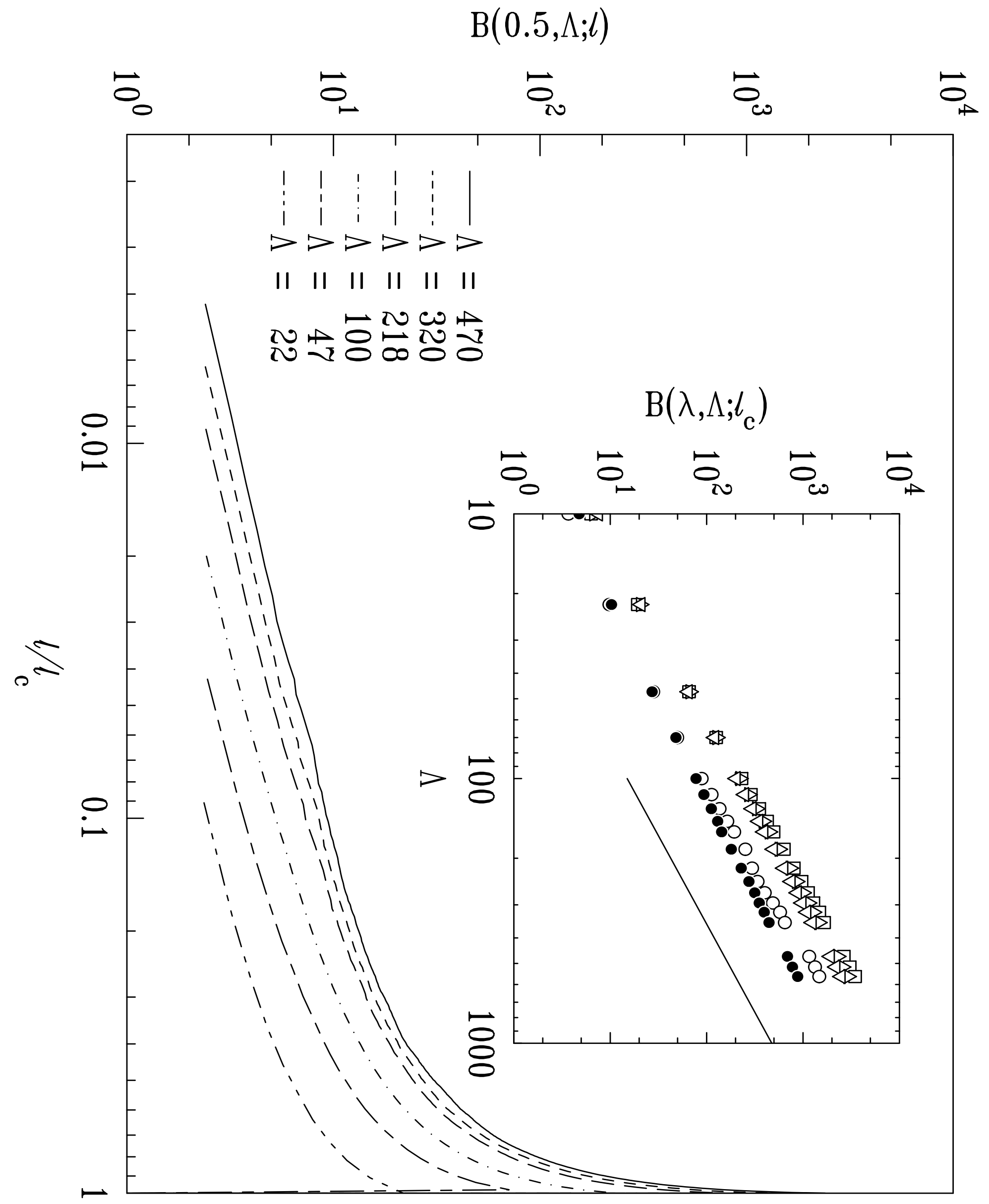



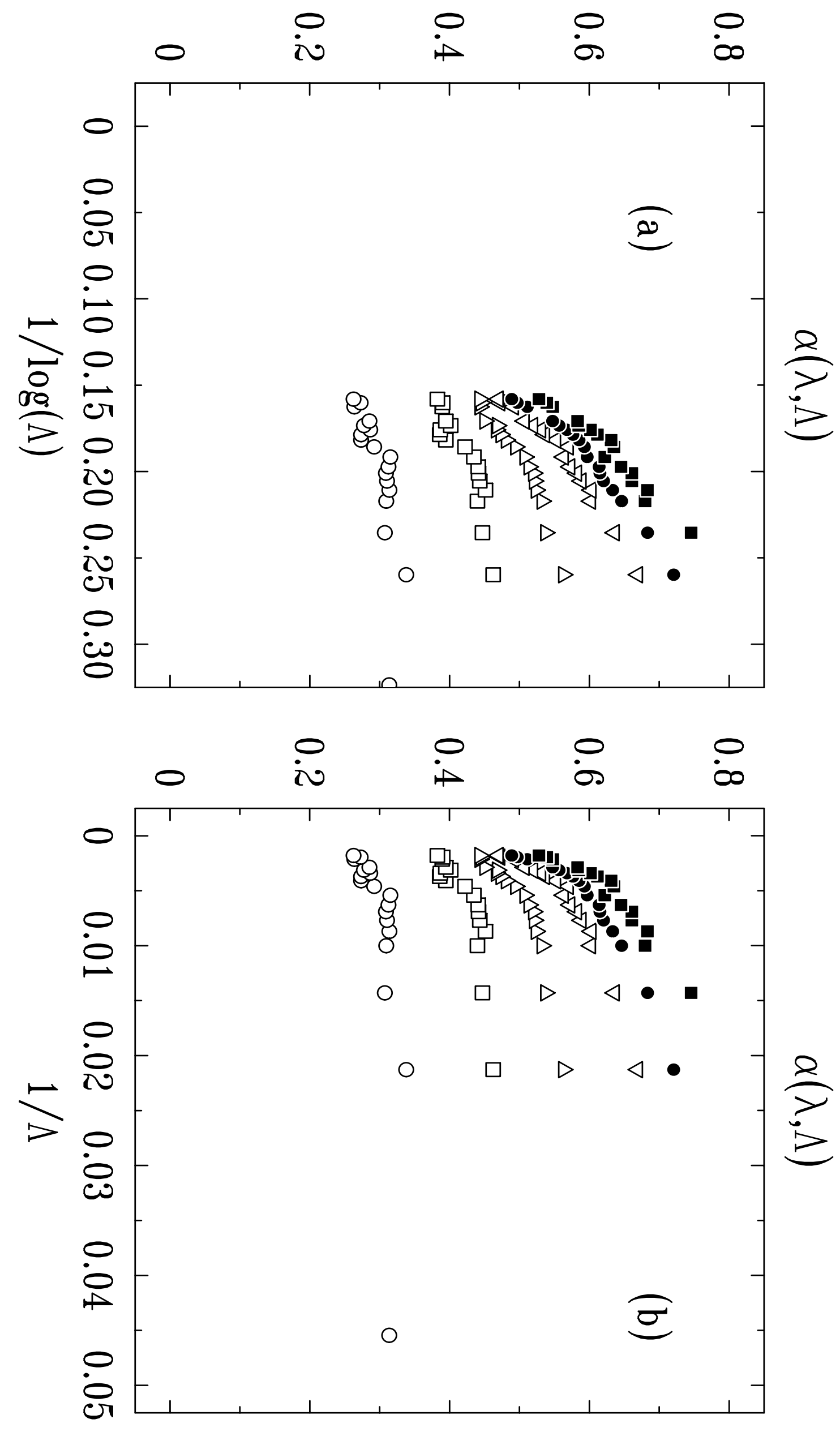


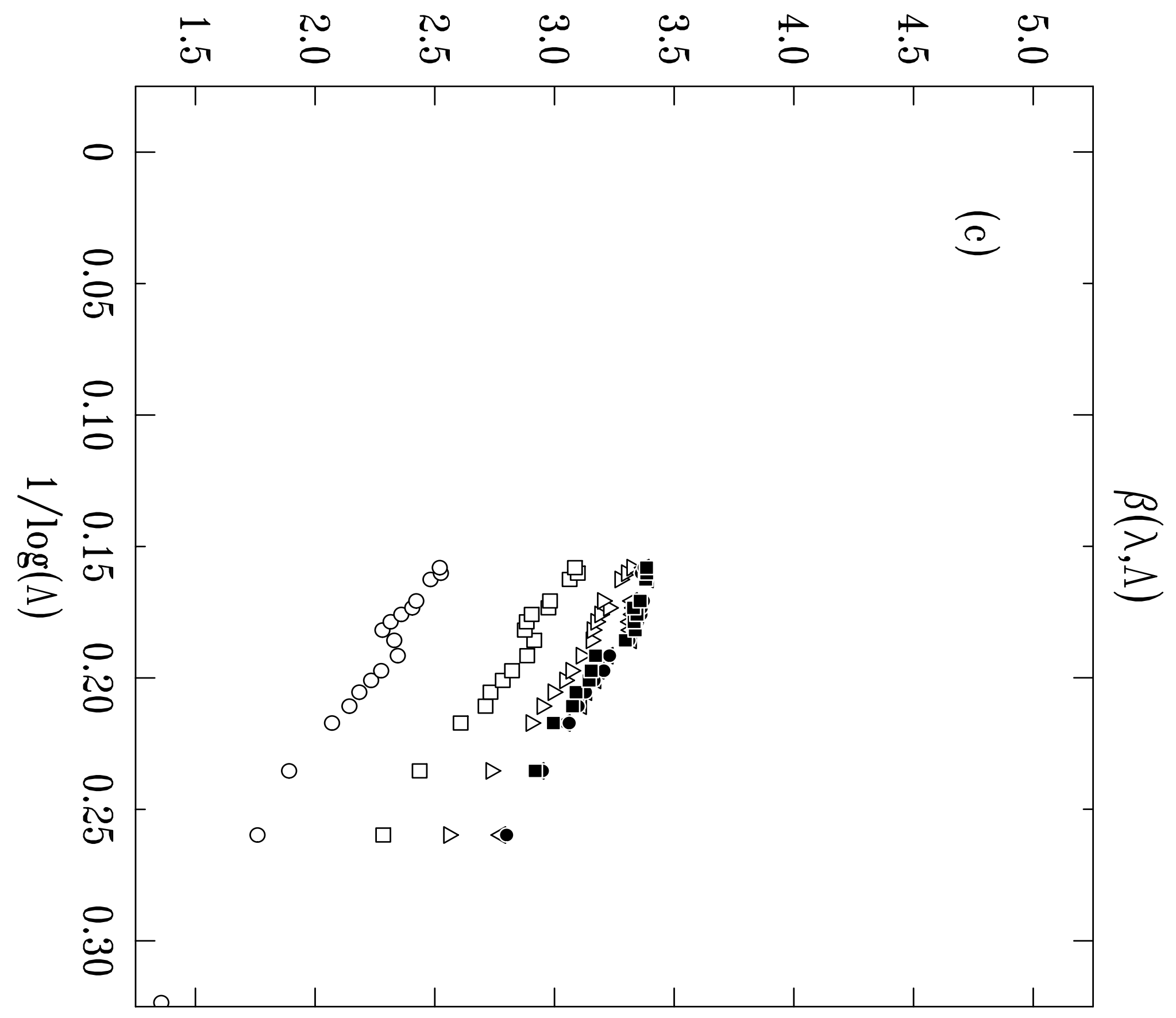




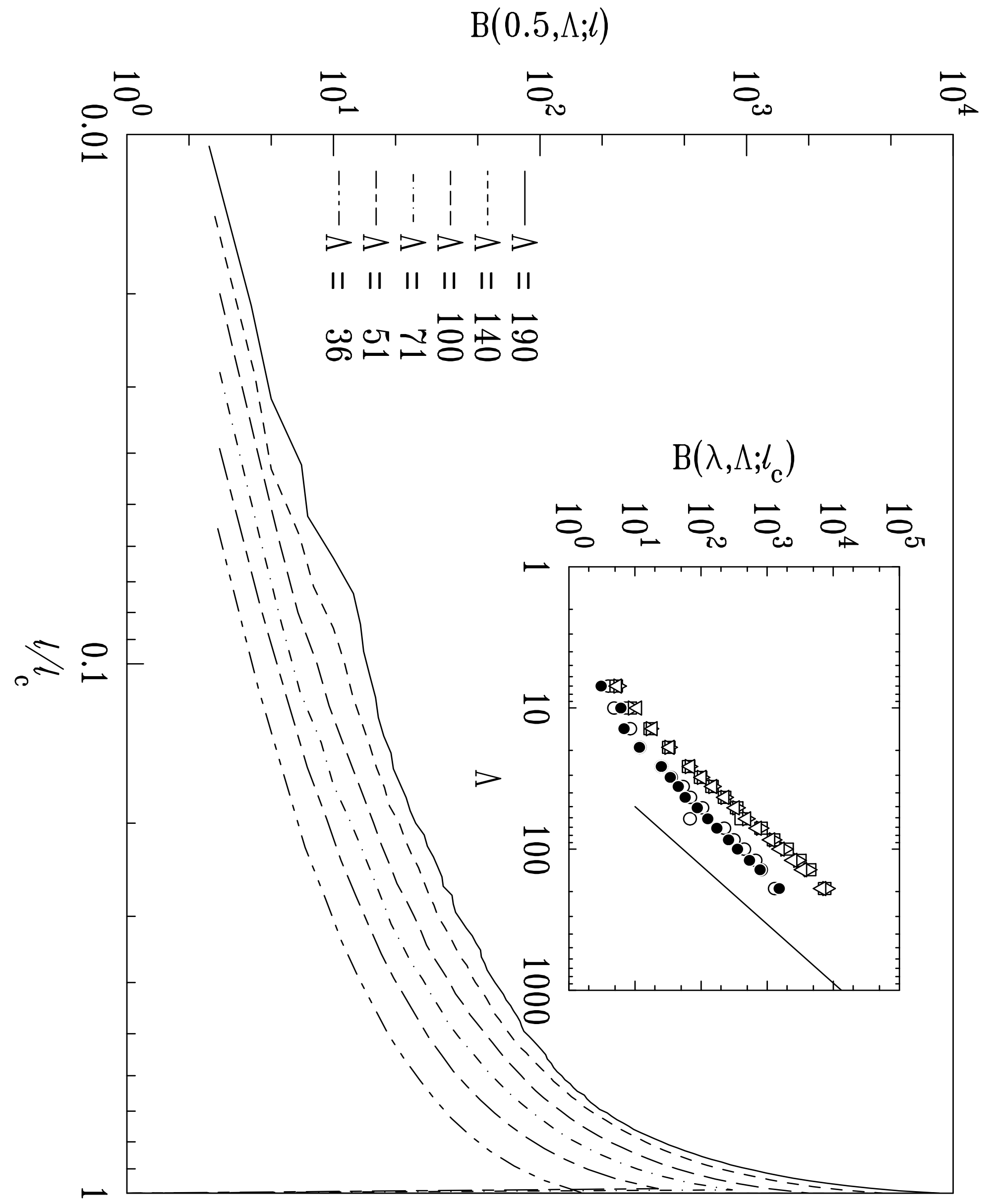




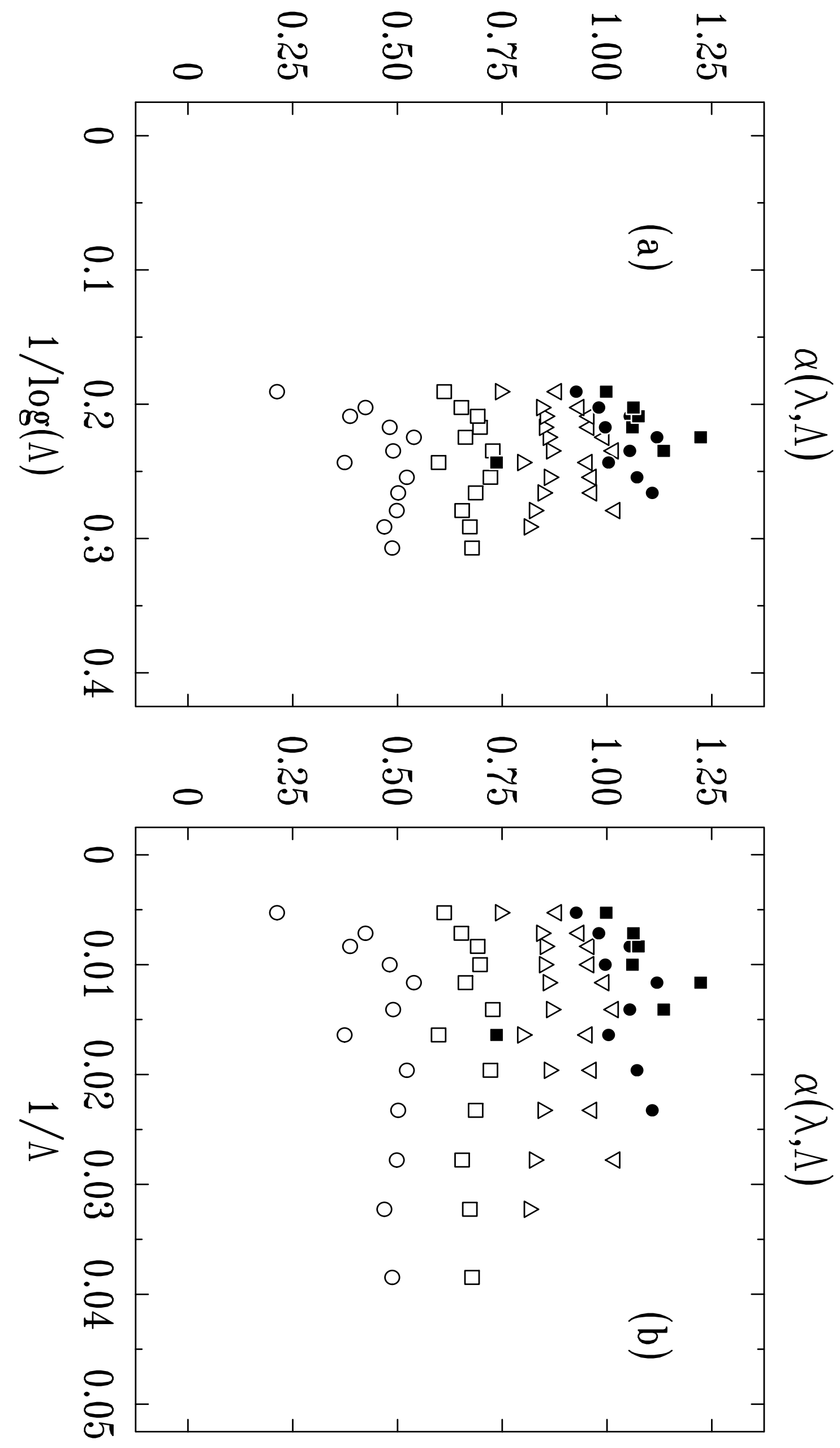




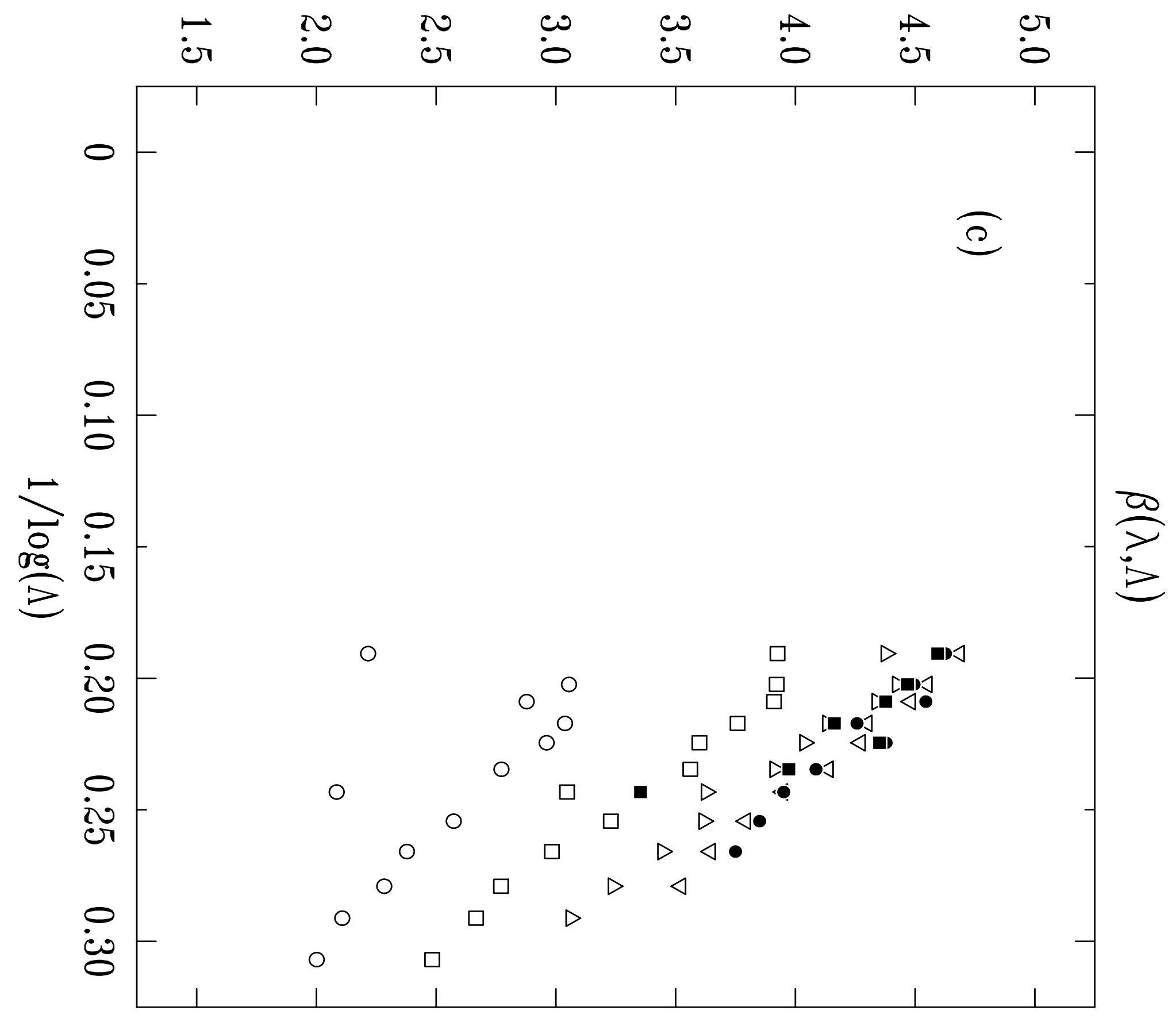

